
Cahiers
de a Recherche
surles Droits
Fondamentaux

\section{Cahiers de la recherche sur les droits} fondamentaux

$13 \mid 2015$

Le droit d'asile

\title{
Rétention des migrants et mesures d'éloignement. Lumières et ombres portées par la CJUE sur la directive « Retour »
}

Detention of Migrants and Return Orders: Clarification and Ambiguity Brought by the CJUE on the "Return" Directive

\section{Marie-Laure Basilien-Gainche}

\section{OpenEdition \\ Journals}

Édition électronique

URL : http://journals.openedition.org/crdf/1218

DOI : $10.4000 /$ crdf. 1218

ISSN : 2264-1246

Éditeur

Presses universitaires de Caen

Édition imprimée

Date de publication : 1 novembre 2015

Pagination : 89-102

ISBN : 978-2-84133-742-2

ISSN : $1634-8842$

\section{Référence électronique}

Marie-Laure Basilien-Gainche, « Rétention des migrants et mesures d'éloignement. Lumières et ombres portées par la CJUE sur la directive « Retour » ", Cahiers de la recherche sur les droits fondamentaux [En ligne], 13 | 2015, mis en ligne le 01 novembre 2016, consulté le 11 février 2020. URL : http://journals.openedition.org/crdf/1218; DOI : 10.4000/crdf.1218 


\title{
Rétention des migrants et mesures d'éloignement. Lumières et ombres portées par la CJUE sur la directive " Retour " ${ }^{1}$
}

\author{
Marie-Laure BASILIEN-GAINCHE \\ Professeure de droit public à l'université Jean Moulin - Lyon 3 \\ Membre de l'Institut universitaire de France
}

\begin{abstract}
I. Le recours à la rétention aux fins d'éloignement. La normalité de l'exceptionnel
A. Les motifs justifiant la rétention aux fins d'éloignement. Des raisons indéfinies

B. Les conditions de la rétention aux fins d'éloignement. Des exigences limitées

II. Le contrôle de la rétention aux fins d'éloignement. La vacuité de la plénitude

A. La nature du contrôle de la rétention aux fins d'éloignement. Des traits équivoques

B. Le terme de la rétention aux fins d'éloignement. Des limbes illégitimes
\end{abstract}

Le Réseau européen des migrations (REM) a publié en novembre 2014 une étude sur le recours à la rétention ${ }^{2}$ et le développement de ses alternatives comme mesures de gestion de l'immigration par vingt-six États européens ${ }^{3}$.
Ce sont quelque 92575 ressortissants de pays tiers (RPT) qui ont été à ce titre détenus en 2013 dans vingt-trois des pays considérés (Chypre, Grèce et Portugal n'ayant pas transmis de données chiffrées) ${ }^{4}$. Si les informations

1. Les réflexions présentées dans cet article ont été précédemment exposées en anglais dans un article paru dans le European Journal of Migration and Law sous le titre «Immigration Detention under the Return Directive: The CJEU Shadowed Lights", European Journal for Migration and Law, no 17,2015, p. 107-129.

2. Le terme «rétention» est celui employé par le droit français pour désigner la privation de liberté des étrangers en situation irrégulière, par contraste au terme «détention» qui renvoie à la privation de liberté des personnes placées dans un établissement pénitentiaire pour y purger une condamnation pénale ou pour y attendre le prononcé de leur jugement. Dans les autres États membres de l'Union européenne, la distinction n'apparaît pas toujours dans le vocabulaire (le même terme "detention» désigne les deux types de privation de liberté au Royaume-Uni) ou dans la pratique (les migrants peuvent être retenus dans les centres de détention que sont les prisons en Allemagne). Il n'en demeure pas moins que la différence entre « rétention» et «détention» est bien ténue qui se résume à une consonne. Voir F. Julien-Laferrière, «La rétention des étrangers aux frontières françaises", Cultures \& Conflits, $\mathrm{n}^{\circ} 23,1996, \mathrm{p} .7-43$.

3. REM, The Use of Detention and Alternatives to Detention in the Context of Immigration Policies, novembre 2014. Cette étude est la synthèse des rapports nationaux qui ont été réalisés concernant vingt-cinq États membres (Allemagne, Autriche, Belgique, Bulgarie, Chypre, Croatie, Espagne, Estonie, Finlande, France, Grèce, Hongrie, Irlande, Lettonie, Lituanie, Luxembourg, Malte, Pays-Bas, Pologne, Portugal, République tchèque, Royaume-Uni, Slovaquie, Slovénie, Suède) et un État associé à l'espace Schengen (Norvège). Le Danemark, l'Italie et la Roumanie sont les trois États membres de l'Union qui n'apparaissent pas dans cette étude. Pour une présentation de cette dernière, voir I. Majcher, "Immigration Detention in Europe: What Are the Facts? A New European Migration Network Study", EU Law Analysis, 5 décembre 2014, en ligne: http://eulawanalysis.blogspot.fr/2014/12/immigration-detention-in-europe-what.html.

4. Les écarts entre les différents États européens sont à remarquer : 38266 RPT ont été placés en rétention en 2013 en France (ce nombre incluant les rétentions opérées dans les territoires d'outre-mer, en particulier la Guyane et Mayotte); 9020 en Espagne; 6496 en Hongrie; 6285 en Belgique ; 243 en Lituanie; 221 en Lettonie; 204 en Slovaquie; 94 en Estonie. Or les variations dans le temps sont, elles aussi, marquantes: entre 2009 et 2013, la Hongrie et la Bulgarie ont enregistré une hausse du nombre de migrants détenus de respectivement $226 \%$ et $600 \%$, alors même que les Pays-Bas et la Slovaquie ont observé une baisse de respectivement $53 \%$ et $65 \%$. 
présentées par le REM se révèlent intéressantes dans leur globalité, elles s'avèrent décevantes par leur imprécision: seuls neuf États ont indiqué le nombre de migrants détenus qui étaient des demandeurs d'asile; seuls cinq États ont renseigné le nombre de RPT détenus qui faisaient l'objet de mesures d'éloignement. Or, à l'échelle de l'Union européenne, la rétention des migrants est employée ${ }^{5}$, et ce dans deux circonstances qu'il convient de distinguer : premièrement, peut être détenu le migrant sollicitant la protection internationale dans le cadre de son accueil sur le territoire de l'État de destination ${ }^{6}$, de l'examen de sa demande d'asile ${ }^{7}$, de son transfert vers l'État membre responsable de l'examen de sa demande en application du règlement Dublin ${ }^{8}$; deuxièmement, peut être détenu le RPT en situation irrégulière faisant l'objet d'une mesure d'éloignement en application de la directive 2008/115/CE du Parlement européen et du Conseil du 16 décembre 2008 relative aux normes et procédures communes applicables dans les États membres au retour des ressortissants de pays tiers en séjour irrégulier ${ }^{9}$. L'article $15, \S 1$, de la directive «Retour " permet en effet aux États membres de «placer en rétention le ressortissant d'un pays tiers qui fait l'objet de procédures de retour afin de préparer le retour et / ou de procéder à l'éloignement ", tout en précisant immédiatement que «toute rétention est aussi brève que possible et n'est maintenue qu'aussi longtemps que le dispositif d'éloignement est en cours et exécuté avec toute la diligence requise».

Certes, ainsi que la définit le REM dans son étude, la rétention des migrants en général et la rétention aux fins d'éloignement en particulier sont des mesures administratives. Il n'en demeure pas moins qu'elles doivent être conçues et employées de manière exceptionnelle, en ce qu'elles emportent une privation de liberté ${ }^{10}$. C'est pourquoi elles sont à utiliser en dernier recours, les principes de nécessité et de proportionnalité s'appliquant pleinement. Il s'agit en effet de prévenir une détention arbitraire $^{11}$, qui est explicitement prohibée par le Pacte des droits civils et politiques (art. 9, \$1) et par la Convention européenne de sauvegarde des droits de l'homme et des libertés fondamentales (Convention EDH; art. 5, § 1). La possibilité pour les États de détenir les migrants ne peut donc qu'être accompagnée de l'affirmation de règles substantielles et procédurales à même de garantir effectivement les droits des migrants ${ }^{12}$, afin d'assurer le respect par les États de leurs obligations internationales induites par la Convention de Genève relative au statut des réfugiés de 1951 pour les RPT sollicitant la protection internationale, et de celles induites par la Convention EDH de 1950 pour tous les migrants y compris ceux en situation irrégulière. Toutefois, il convient de souligner que l'article 5 , $\$ 1$, de la Convention EDH ne se contente pas d'affirmer que «toute personne a droit à la liberté et à la sûreté» : en effet il ajoute que «nul ne peut être privé de sa liberté, sauf dans les cas suivants et selon les voies légales », parmi lesquels figure un point f) qui nous intéresse ici grandement puisqu'il fait état «de l'arrestation ou de la détention régulières d'une personne pour l'empêcher de pénétrer irrégulièrement dans le territoire, ou contre laquelle une procédure d'expulsion ou d'extradition est en cours ».

Depuis sa décision dans l'affaire Chahal, la Cour de Strasbourg (CJUE) a constamment interprété cette disposition permettant la rétention pour lutter contre l'immigration irrégulière d'une manière qu'on pourrait souhaiter plus stricte. En effet, elle affirme que «cette disposition (art. 5-1-f) n'exige pas que la détention d'une personne contre laquelle une procédure d'expulsion est en cours soit considérée comme raisonnablement nécessaire, par exemple pour l'empêcher de commettre une infraction ou de s'enfuir ${ }^{13}$. Certes, la Cour européenne des droits de l'homme insiste sur le fait que, pour être légale, la rétention des migrants aux fins de lutte contre l'immigration irrégulière ne peut avoir lieu que si une perspective raisonnable d'éloignement existe et que si

5. Concernant le recours à la rétention dans les politiques d'immigration, voir S. Silverman, E. Massa, "Why Immigration Detention is Unique», Population, Space and Place, vol. 18, n 6, 2012, p. 677-686; M. Bosworth, Inside Immigration Detention, Oxford - New York, Oxford University Press, 2014; M. Ceccorulli, N. Labanca, The EU, Migration and the Politics of Administrative Detention, Londres - New York, Routledge, 2014; D. Wilsher, Immigration Detention: Law, History, Politics, Cambridge - New York, Cambridge University Press, 2012.

6. Directive $2003 / 9 / C E$ du Conseil du 27 janvier 2003 relative à des normes minimales pour l'accueil des demandeurs d'asile dans les États membres (art. 14), et sa refonte par la directive 2013/33/UE du Parlement européen et du Conseil du 26 juin 2013 (art. 8 à 11).

7. Directive $2005 / 85 / \mathrm{CE}$ du Conseil du $1^{\text {er }}$ décembre 2005 relative à des normes minimales concernant la procédure d'octroi et de retrait du statut de réfugié dans les États membres (art. 18), et sa refonte par la directive 2013/32/EU du Parlement européen et du Conseil du 26 juin 2013 (art. 26).

8. Règlement $(\mathrm{CE}) \mathrm{n}^{\circ} 343 / 2003$ du Conseil du 18 février 2003 établissant les critères et mécanismes de détermination de l'État membre responsable de l'examen d'une demande d'asile présentée dans l'un des États membres par un ressortissant d'un pays tiers (art. 17), et sa refonte par le règlement (UE) no 604/2013 du Parlement européen et du Conseil du 26 juin 2013 (art. 28).

9. Pour une analyse de la directive «Retour», voir entre autres K. Parrot, C. Santulli, «La "directive retour", l’Union européenne contre les étrangers», Revue critique de droit international privé, vol. 98, n², 2009, p. 205-249; A. Baldaccini, "The Return and Removal of Irregular Migrants under EU Law: An Analysis of the Returns Directive», European Journal of Migration and Law, vol. 11, no 1, 2009, p. 1-17; The Returns Directive: Central Themes, Problem Issues, and Implementation in Selected Member States, K. Zwaan (dir.), Nimègue, Wolf Legal Publishers, 2011; D. Acosta Arcarazo, "The Returns Directive», in EU Immigration and Asylum Law, vol. II, EU Immigration Law, S. Peers, E. Guild, D. Acosta Arcarazo, K. Groenendijk, V. Moreno-Lax (dir.), Leyde, Brill, 2012, p. 483-523.

10. Voir Nations unies, "Détention d'immigrants en situation irrégulière», in Rapport du Groupe de travail sur la détention arbitraire, 18 janvier 2010, A/HCR/13/30, \$54-65; Nations unies, «L'interdiction de la privation arbitraire de liberté en droit international», in Rapport du Groupe de travail sur la détention arbitraire, 24 décembre 2012, A/HCR/22/44, \$42-51.

11. Comité des droits de l'homme, 30 avril 1997, A. c. Australie, 560/1993, $\$ 9.2 ; 13$ novembre 2006, Danyal Shafiq c. Australie, 1324/2004, $\$ 7.2$.

12. Cour EDH, 25 juin 1996, Amuur c. France, nº 19776/92.

13. Cour EDH, 15 novembre 1996, Chahal c. Royaume-Uni, no 22414/93, \$112. 
les procédures sont conduites avec toute la diligence requise ${ }^{14}$. Mais elle se refuse malheureusement à mener l'examen du respect d'une condition de nécessité: elle exerce donc un contrôle hélas limité sur l'emploi de la rétention aux fins d'éloignement et laisse ainsi aux États membres une large marge d'appréciation. La raison d'une telle position est clairement exposée par la Cour: dans sa décision dans l'affaire Amuur, elle affirme que les États jouissent du «droit indéniable de contrôler souverainement l'entrée et le séjour des étrangers sur leur territoire ${ }^{15}$; puis dans sa décision dans l'affaire Saadi, elle soutient que «la faculté pour les États de placer en détention des candidats à l'immigration ayant sollicité - par le biais d'une demande d'asile ou non - l'autorisation d'entrer dans le pays est un corollaire indispensable de ce droit ${ }^{16}$. Il en résulte que la règle de droit semble moins stricte, plus souple en matière migratoire ${ }^{17}$, que la protection des droits semble moins forte, plus lâche pour les migrants ${ }^{18}$. Les prérogatives étatiques afférentes au contrôle des frontières paraissent prévaloir alors, expliquant pourquoi la rétention des migrants en général et des migrants sous le coup de mesures d'éloignement en particulier se soit tant développée en Europe.

Le recours à la rétention en matière d'immigration soulève donc de sérieuses problématiques, qui ont constamment fait l'objet de questionnements et de critiques $^{19}$, et qui ont pris de nos jours une dimension pour le moins sensible. Alors que l'Union européenne a adopté la directive 2008/115/CE entrée en vigueur dans le contexte institutionnel de l'après-Lisbonne ${ }^{20}$, on peut se demander si la CJUE - dont la compétence a été ainsi étendue aux affaires d'immigration et d'asile - offre désormais plus de garanties que la Cour européenne des droits de l'homme aux RPT privés de liberté. La Cour de Luxembourg a rendu plusieurs décisions qui ont permis d'apporter quelques précisions sur l'interprétation à donner de diverses dispositions de la directive «Retour», si bien qu'une appréciation de sa position peut en être proposée. Si la CJUE a parfois rendu des arrêts exposant des positions claires et nettes, elle a aussi pris des décisions si nuancées et si balancées que des ambiguiités et des incertitudes sont apparues concernant la réelle effectivité de la protection des droits fondamentaux des migrants objets de mesures d'éloignement. Dans sa décision Filev et Osmani, la Cour a interprété en des termes fermes et limpides la disposition de la directive concernant l'interdiction d'entrée sur le territoire:

[...] l'article 11, paragraphe 2, de la directive 2008/115 s'oppose au maintien des effets d'interdictions d'entrée ayant une durée illimitée imposées avant la date d'applicabilité de la directive 2008/115, telles que celles en cause au principal, au-delà de la durée maximale d'interdiction prévue à cette disposition, sauf si ces interdictions d'entrée ont été prononcées à l'encontre de ressortissants de pays tiers constituant une menace grave pour l'ordre public, la sécurité publique ou la sécurité nationale ${ }^{21}$.

Il n'en demeure pas moins que les autres décisions rendues par le juge de l'Union européenne sur la directive «Retour» en général et sur la rétention des migrants aux fins d'éloignement en particulier apparaissent nettement moins certaines et moins assurées, plus confuses et douteuses. Certes la Cour se réfère fréquemment à la Charte des droits fondamentaux. Cependant, faute de donner des réponses claires aux questions à elle posées, elle laisse de nombreuses zones d'incertitudes dans l'ombre, les livrant en conséquence à l'appréciation des juridictions nationales, quand ce n'est pas à la discrétion des États membres. Quels sont les motifs qui permettent de justifier le recours à la rétention aux fins d'éloignement d'un RPT en situation irrégulière? Et quelles sont les conditions à remplir pour qu'une telle privation de liberté soit en conformité avec les normes de l'Union? Quelle est l'intensité du contrôle juridictionnel exercé par les juges nationaux sur les mesures de rétention des migrants faisant l'objet de mesures de retour? Et quelles conséquences ces décisions juridictionnelles

14. Cour EDH, 22 mars 1995, Quinn c. France, $\mathrm{n}^{\circ}$ 18580/91, \$ 48; 25 janvier 2005, Singh c. République Tchèque, $\mathrm{n}^{\circ} 60538 / 00, \S 61$.

15. Cour EDH, 25 juin 1996, Amuur c. France, $\$ 41$.

16. Cour EDH, GC, 29 janvier 2008, Saadi c. Royaume-Uni, no 13229/03, \$ 64. L’opinion en partie dissidente commune aux juges Rozakis, Tulkens, Kovler, Hajiyev, Spielmann, et Hirvelä doit être relevée ici. Elle ne se contente pas de critiquer sévèrement l'arrêt en ce qu'il «n'hésite donc pas à opérer un amalgame complet entre toutes les catégories d'étrangers et toutes les situations qui sont les leurs - immigrés clandestins, personnes susceptibles d'être expulsées et celles qui ont commis des infractions -, en les incluant sans nuance dans le contrôle général de l'immigration qui relève de la souveraineté sans limite des États». Elle pose sans ambages une question essentielle: "peut-on aussi accepter aujourd'hui que l'article 5 de la Convention qui a joué un rôle majeur dans le contrôle de l'arbitraire en matière de privation de liberté fournisse un niveau de protection moindre dans le domaine de l'asile et de l'immigration qui sont, socialement et humainement, les questions les plus critiques de ces prochaines années?». Et elle conclut avec force: «Être étranger est-il un crime? Nous ne le pensons pas» (Cour EDH, GC, 29 janvier 2008, Saadi c. Royaume-Uni; nous soulignons).

17. Voir M. Fordham QC, J. N. Stefanelli, S. Eser, Immigration Detention and the Rule of Law. Safeguarding Principles, Londres, Bingham Institute for the Rule of Law, 2013.

18. C. Costello, «Human Rights and the Elusive Universal Subject. Immigration Detention under International Human Rights and EU Law», Indiana Journal of Global Legal Studies, vol. 19, $\mathrm{n}^{\circ}$ 1, 2012, p. 257-303.

19. Étude STEPS Consulting Social pour le Parlement européen, Conditions des ressortissants de pays tiers retenus dans des centres (camps de détention, centres ouverts, ainsi que des zones de transit), avec une attention particulière portée aux services et moyens en faveur des personnes aux besoins spécifiques au sein des 25 Etats membres de l'Union européenne, décembre 2007; Conseil de l'Europe, Assemblée parlementaire, Commission des migrations, des réfugiés et de la population, La rétention administrative des demandeurs d'asile et des migrants en situation irrégulière en Europe, doc. 12105, 11 janvier 2010. Voir aussi L. L. Martin, M. L. Mitchelson, « Geographies of Detention and Imprisonment: Interrogating Spatial Practices of Confinement, Discipline, Law, and State Power", Geography Compass, vol. 3, n 1, 2009, p. 459-477.

20. Voir M.-L. Basilien-Gainche, "The EU Immigration and Asylum Policy in the Post-Lisbon Institutional Context», in The Treaty of Lisbon and the Future of European Law and Policy, L. Rubini, M. Trybus (dir.), Londres, Edward Elgar Publishing, 2012, p. $355-378$.

21. CJUE, 19 septembre 2013, Filev et Osmani, aff. C-297/12, pt 44. 
peuvent-elles emporter? Si la Cour de Luxembourg a été conduite à aborder les questions du recours aux mesures de rétention aux fins d'éloignement de RPT en situation irrégulière (I) et du contrôle dont elles doivent faire l'objet (II), les éclairages qu'elle a donnés sur les dispositions de la directive 2008/115/CE ont maintenu des incertitudes, voire créé des ambiguïtés, autant d'ombres portées qui ne sont pas sans poser problème.

\section{Le recours à la rétention aux fins d'éloignement. La normalité de l'exceptionnel}

En donnant son interprétation de l'article 2 de la directive «Retour» dans son arrêt Arslan, la CJUE en a clarifié le champ d'application: à ses yeux, ce texte

[...] n'est pas applicable à un ressortissant de pays tiers qui a introduit une demande de protection internationale, au sens de la directive $2005 / 85$, et ce pendant la période courant de l'introduction de ladite demande jusqu'à l'adoption de la décision de premier ressort statuant sur cette demande ou, le cas échéant, jusqu'à l'issue du recours qui aurait été introduit contre ladite décision ${ }^{22}$.

La Cour souligne ainsi que le seul fait pour un RPT de déposer une demande d'asile, quand bien même cela a lieu durant les procédures d'éloignement, ne saurait justifier sa rétention. Deux régimes de la rétention des migrants coexistent en effet qui se chevauchent, celui rattaché à la demande de protection internationale d'une $\operatorname{part}^{23}$ et celui correspondant aux procédures de retour des RPT en situation irrégulière d'autre part ${ }^{24}$. Or, pour François Crépeau, rapporteur spécial sur les droits de l'homme des migrants, les mesures de rétention en matière d'immigration devraient être employées seulement en dernier ressort et pour une durée la plus courte possible, et devraient être utilisées seulement quand il n'y a pas de mesures moins coercitives qui puissent être adoptées permettant à l'État d'atteindre ses objectifs : les États ont l'obligation d'établir une présomption en faveur de la liberté dans leur droit national, en développant les alternatives à la rétention, en procédant à une évaluation au cas par cas, et en recourant aux mesures les moins intrusives et restrictives possibles ${ }^{25}$. Tel semble être d'ailleurs la perspective adoptée par la directive 2008/115/CE puisque son article 15, $\$ 1$, permet aux États membres de recourir à la rétention «à moins que d'autres mesures suffisantes, mais moins coercitives, puissent être appliquées efficacement dans un cas particulier».

Quelles sont les alternatives à la rétention que l'on observe le plus couramment en Europe ? L'assignation à résidence, l'enregistrement ou dépôt de documents, l'obligation de rapports, le dépôt de cautions ou de garanties, indique l'étude du REM qui ne dit cependant pas si ces alternatives prévues dans les législations nationales des États examinés sont effectivement employées ou non ${ }^{26}$. Dans sa communication sur la politique européenne de retour $^{27}$, la Commission donne des informations sur ce point: sur les quatre-vingt-sept bases légales qui prévoient des alternatives à la rétention dans les législations nationales de trente et un États considérés, seuls $32 \%$ ont été effectivement utilisées, révélant le fossé existant en la matière entre les textes et les pratiques. Bien plus, certains ordres juridiques nationaux semblent toujours regarder la rétention comme la norme et les alternatives comme des exceptions, mettant en évidence que les mesures les moins coercitives sont loin encore de prévaloir en Europe $^{28}$. Les énoncés incertains de la directive « Retour» paraissent moins imposer aux États membres d'instaurer des alternatives à la rétention, que leur offrir la possibilité d'opter pour des mesures moins coercitives. Bien que le considérant 16 de la directive $2008 / 115 /$ CE pose que «le recours à la rétention aux fins d'éloignement devrait être limité et subordonné au respect du principe de proportionnalité en ce qui concerne les moyens utilisés et les objectifs poursuivis » et que «la rétention n'est justifiée que pour préparer le retour ou procéder à l'éloignement et si l'application de mesures moins coercitives ne suffirait pas ", la pratique de la rétention des migrants aux fins d'éloignement semble bel et bien normalisée. Malheureusement, les précisions données par la CJUE concernant les motifs (A) et les conditions (B) de la rétention des migrants dans le cadre de la directive « Retour» se révèlent insuffisantes pour imposer que cet outil qui devrait être exceptionnel ne demeure une mesure habituelle.

\section{A. Les motifs justifiant la rétention aux fins d'éloignement. Des raisons indéfinies}

Certes l'esprit de la directive 2008/115/CE semble bien consister à limiter le recours à la rétention, d'une part en le soumettant au principe de proportionnalité, d'autre part

22. CJUE, 30 mai 2013, Arslan, aff. C-534/11, pt 49

23. Directive 2003/9/CE, modifiée par la directive 2013/33/UE; directive 2005/85/CE, modifiée par la directive 2013/32/EU.

24. Directive $2008 / 115 / C E$.

25. F. Crépeau, "Détention de migrants en situation irrégulière» et "Conclusions et recommandations", in Rapport du rapporteur spécial sur les droits de l'homme des migrants, $\mathrm{A} / \mathrm{HRC} / 2 \mathrm{O} / 24,2$ avril 2012, $\$ 68$.

26. REM, The Use of Detention and Alternatives to Detention...

27. Communication de la Commission au Conseil et au Parlement européen sur la politique de l'Union européenne en matière de retour, 28 mars 2014, $\operatorname{COM}(2014) 199$.

28. Art. 77 de la loi autrichienne FLG I no 100/2005 amendé par FLG I no 157/2005, FLG I nº 4/2008 et FLG I nº 29/2009; section 36 de la loi danoise sur les étrangers $n^{\circ} 947$ du 24 août 2011 ; art. 90 et 91 de la loi polonaise $n^{\circ} 128$ du 13 juin 2003 qui a été plusieurs fois modifiée depuis son adoption; art. 551-1 et 551-4 du Code de l'entrée et du séjour des étrangers et du droit d'asile. 
en établissant des garanties minimales pour les migrants détenus. Pourtant, la lettre de ce texte paraît avoir échoué à insuffler un tel esprit dans les législations nationales, qui pour nombre d'entre elles ne considèrent pas la rétention comme une mesure à employer de manière exceptionnelle, et qui pour certaines d'entre elles ne préviennent pas la possibilité de détentions arbitraires. Comme le met en évidence Izabella Majcher, la directive est dans l'incapacité de circonscrire efficacement le recours à la rétention, faute de définir clairement les motifs qui peuvent en justifier l'emploi ${ }^{29}$. D'abord, la directive « Retour» n'énumère pas de façon exhaustive les fondements sur la base desquels peut être décidée une rétention aux fins d'éloignement; ensuite, elle ne demande pas aux États membres de le faire dans leur droit national. Or la liste des motifs qui sont le plus souvent admis par les législations nationales des États européens pour justifier la rétention aux fins d'éloignement d'un RPT en situation irrégulière est révélatrice de la tendance à envisager et à organiser un emploi habituel de la privation de liberté. Elle est en effet prévue quand le migrant présente un risque de fuite (vingt-deux États), cherche à éviter les procédures d'éloignement (vingt États), tend à empêcher la réalisation des mesures de retour (quatorze États), ne remplit pas les conditions permettant d'utiliser les alternatives à la rétention (douze États), constitue une menace pour la sécurité nationale et l'ordre public (douze États), n'a pas procédé à son retour dans le délai de départ volontaire imparti (onze États), se prévaut d'une identité qu'il convient de vérifier (onze États), conduit les autorités nationales à raisonnablement penser qu'il pourrait commettre des infractions (six États) ${ }^{30}$. Or, seuls les deux premiers fondements sont explicitement prévus par la directive 2008/115/CE: un RPT en situation irrégulière peut être détenu quand il existe un risque de fuite (art. 15, §1, a) ou quand il cherche à éviter ou empêcher la préparation du retour ou la procédure d'éloignement ( $\operatorname{art} .15, \$ 1, b$ ).

C'est tout d'abord la référence au «risque de fuite» qui inquiète. L'article $3, \$ 7$, de la directive 2008/115/CE le définit comme désignant «le fait qu'il existe des raisons, dans un cas particulier et sur la base de critères objectifs définis par la loi, de penser qu'un ressortissant d'un pays tiers faisant l'objet de procédures de retour peut prendre la fuite». Aucune explication n'étant donnée concernant ce que peuvent être les «critères objectifs » à prendre en compte, une large marge d'appréciation est laissée aux États membres dans l'interprétation de la disposition. L'exemple de la législation française est frappant: à la lecture de l'article 511-3-1 du Code de l'entrée et du séjour des étrangers et du droit d'asile, il apparaît que le risque de fuite justifiant la rétention d'un migrant en situation irrégulière (et le refus de lui accorder un délai de départ volontaire pour organiser son retour) renvoie à neuf situations différentes, dont six sont sans aucun doute non conformes à la norme européenne ${ }^{31}$. Il n'est guère utile d'insister davantage sur le fait que, dans un tel contexte, les décisions rendues par la Cour de Luxembourg revêtent une importance toute particulière. Si la CJUE a indiqué dans l'affaire Sagor ${ }^{32}$ que "toute appréciation [du risque de fuite] doit se fonder sur un examen individuel du cas de l'intéressé», l'apport est en réalité limité qui s'avère insuffisant pour contraindre et donc contrer les pratiques que les États membres ont développées pour exploiter la notion de risque de fuite. Opportunément, la Cour a eu l'occasion dans l'affaire $M a h d i^{33}$ de préciser ce que le risque de fuite peut être ou non: il est clairement affirmé que le fait pour un RPT en situation irrégulière de ne pas disposer d'un document d'identité valide ne saurait constituer en soi un risque de fuite et ne saurait donc justifier une rétention aux fins d'éloignement. Néanmoins, la position que la Cour a adoptée s'avère au final décevante, dans la mesure où elle laisse au juge de renvoi la possibilité de prendre en compte un tel élément pour apprécier la légalité du recours par les autorités nationales à une telle rétention. C'est donc avec impatience et inquiétude qu'était attendue la réponse que la Cour donnerait à la question préjudicielle posée par le Raad van State (Pays-Bas) dans l'affaire $Z$. $Z h$. Mais le juge de Luxembourg a de nouveau déçu, sa décision ne s'attardant pas sur la notion de risque de fuite et se contentant de renvoyer les États membres à leur marge d'appréciation ${ }^{34}$.

29. I. Majcher, «The European Union Returns Directive: Does it Prevent Arbitrary Detention?», Oxford Monitor of Forced Migration, vol. 3, n 2, 2013, p. 24.

30. REM, The Use of Detention and Alternatives to Detention... L'étude du REM énumère aussi les fondements qui sont le plus souvent prévus par les législations nationales des vingt-six États considérés pour justifier la rétention des demandeurs d'asile: le besoin de vérifier l'identité du RPT considéré (dix-sept États), le risque de fuite (seize États), la menace à l'ordre public ou à la sécurité nationale (quinze États), la suspicion d'un détournement de la procédure de demande de la protection internationale (onze États), la non-satisfaction des conditions posées au recours aux alternatives à la rétention (neuf États), l'absence de documents d'identité (huit États), et la raison de croire que le migrant pourrait commettre une infraction (sept États). Qu'il nous soit permis de souligner ici que nombre des motifs admis par les normes européennes pour justifier la rétention des demandeurs d'asile sont hautement problématiques: art. 15, \$ 1, de la directive «Retour»; art. 28 du règlement n 604/2013 du Parlement européen et du Conseil du 26 juin 2013 établissant les critères et mécanismes de détermination de l'État membre responsable de l'examen d'une demande de protection internationale introduite dans l'un des États membres par un ressortissant de pays tiers ou un apatride (refonte); art. 8 de la directive 2013/33/UE du Parlement européen et du Conseil du 26 juin 2013 établissant des normes pour l'accueil des personnes demandant la protection internationale (refonte). En effet, ces dispositions ne semblent pas être en conformité avec le droit international des droits de l'homme en général, et la Convention de Genève de 1951 sur le statut des réfugiés en particulier. Voir M.-L. Basilien-Gainche, «La norme et l'exception. L'effectivité en péril du droit d'asile en Europe», à paraître prochainement dans l'Annuaire du droit de l'Union européenne.

31. Disposition insérée par l'article 39 de la loi n ${ }^{\circ}$ 2011-672 relative à l'immigration, à l'intégration et à la nationalité.

32. CJUE, 6 décembre 2012, Md Sagor, aff. C-430/11, pt 41. Voir M.-L. Basilien-Gainche, «Pénalisation du séjour irrégulier: quand le souci d'effet utile l'emporte sur le respect du droit et des droits», Lettre "Actualités Droits-Libertés» de La revue des droits de l'homme, 12 décembre 2012 , en ligne: http://revdh.org/2012/12/12.

33. CJUE, 5 juin 2014, Bashir Mohamed Ali Mahdi, aff. C-146/14 PPU, pt 69. Voir M.-L. Basilien-Gainche, «Judicial Control of Detention: A Deceptive Upheaval?», EU Law Analysis, 10 juin 2014, en ligne: http://eulawanalysis.blogspot.fr/2014/o6/marie-laure-basilien-gainche.html.

34. CJUE, 11 juin 2015, Z. Zh., aff. C-554/13 
Quant aux motifs justifiant la rétention aux fins d'éloignement qui sont prévus par les législations nationales des États membres sans l'être par la norme européenne, outre qu'ils peuvent paraître contraires à la directive 2008/115/CE et pourraient ainsi faire l'objet de procédures en infraction si tant est que la Commission en ait l'audace et l'ambition, ils sont à questionner. En effet, certains d'entre eux ne sont liés ni à la gestion de l'immigration irrégulière, ni aux exigences des procédures de retour ${ }^{35}$. Ainsi du fondement tiré de la prévention d'une éventuelle infraction ${ }^{36}$, dont on peut se demander s'il ne devrait pas plutôt relever du droit pénal que du droit des migrations. Ainsi encore des dispositions nationales qui autorisent la privation de liberté des migrants en situation irrégulière durant les procédures d'éloignement, afin de protéger l'ordre public $^{37}$, la santé publique ${ }^{38}$, ou la sécurité nationale, ce qui ne semble guère en conformité avec la Convention EDH. Aux yeux de la Cour de Strasbourg, «les alinéas a) à f) de l'article $5 \$ 1$ contiennent une liste exhaustive des motifs autorisant la privation de liberté; pareille mesure n'est pas régulière si elle ne relève pas de l'un de ces motifs ${ }^{39}$. Or l'article $5, \$ 1, \mathrm{f}$, permet que les étrangers soient détenus uniquement dans deux cas seulement: pour prévenir leur entrée irrégulière sur le territoire ou pour mener à bien les actions qui doivent être prises pour le retour. Aussi tout autre motif, y compris ceux renvoyant à la protection de l'ordre public, ne sont-ils pas admissibles. Quoique ce point soit des plus clairs, il a fallu à la Cour de Luxembourg l'affirmer dans son arrêt Kadzoev: «La possibilité de placer une personne en rétention pour des raisons d'ordre public et de sécurité publique ne saurait trouver son fondement dans la directive 2008/115", car de telles raisons ne peuvent constituer en elles-mêmes des motifs justifiant la rétention aux fins d'éloignement quand il n'y a pas de procédures de retour en cours ${ }^{4}$.

Les motifs de décider de la rétention aux fins d'éloignement sont problématiques. Les motifs permettant de prolonger la durée de la rétention le sont aussi. En effet, au titre de l'article $15, \$ 6$, de la directive 2008/115/CE, la rétention aux fins d'éloignement peut voir sa durée initiale de dix-huit mois étendue en cas de défaut de coopération du RPT détenu ou en cas de retards dans l'obtention auprès des autorités des États tiers des documents nécessaires à l'organisation du retour. Le premier de ces motifs pose problème, en ce que le défaut de coopération de la part du RPT existe pratiquement toujours, tant il est vrai que la plupart des migrants manifestent des réticences quand ce ne sont pas des résistances à être éloignés. Certes la Cour de Luxembourg a précisé dans l'arrêt Mahdi que le défaut de coopération peut seulement résulter de l'attitude personnelle du RPT sous le coup d'une mesure de retour ${ }^{41}$. Toutefois, elle a omis de répondre clairement à l'une des questions posées par le juge de renvoi, en regardant le fait pour un migrant détenu de ne pas disposer d'un document d'identité valide non comme une question de droit, mais comme une question de fait. En renvoyant au juge national le soin de trancher la question, la CJUE donne une large marge d'appréciation voire de discrétion aux juridictions nationales pour évaluer les situations de chacun des cas qu'elles ont à connaître. Pour ce qui est du second motif permettant de rallonger la période de rétention aux fins d'éloignement, elle découle des difficultés ou des retards que l'État national du migrant rencontre ou génère dans la délivrance des documents nécessaires à l'organisation de son retour. Justifier ainsi la privation de liberté est en soi préoccupant. Toute rétention devrait être considérée comme illégale dès lors que l'obstacle opposé à la réalisation de l'éloignement ne résulte pas de la sphère d'influence du détenu, pour le groupe de travail des Nations unies sur la détention arbitraire ${ }^{42}$. Alors que la CJUE ne lève pas les incertitudes et inquiétudes au sujet des motifs permettant de décider de rallonger la rétention aux fins d'éloignement - qui peut ainsi durer bien au-delà de ce qui est raisonnable et nécessaire pour procéder au retour -, elle pose des exigences concernant les conditions de la rétention qui se révèlent malheureusement bien relatives.

\section{B. Les conditions de la rétention aux fins d'éloignement. Des exigences limitées}

Si la directive «Retour» limite à une durée de six mois pouvant être étendue à dix-huit mois la rétention des migrants irréguliers en instance d'éloignement ${ }^{43}$, c'est une

35. Agence des droits fondamentaux de l'Union européenne, Rétention des ressortissants de pays tiers dans le cadre des procédures de retour, novembre 2010, p. 15-20.

36. Voir, par exemple, section 121 de la loi finlandaise $n^{\circ} 301 / 2004$, et art. 18 de la loi grecque $n^{\circ} 3907 / 2011$.

37. Voir, par exemple, art. 124 de la loi tchèque $\mathrm{n}^{\circ}$ 326/1999, et section 51 de la loi lettone sur l'immigration.

38. Voir, par exemple, art. 113 de la loi lithuanienne $n^{\circ}$ IX-2206 sur le statut des étrangers.

39. Cour EDH, GC, 29 janvier 2008, Saadi c. Royaume-Uni, $\$ 43 ; 19$ février 2009, A. c. Royaume-Uni, nº 3455/05, \$163.

40. CJCE, 30 novembre 2009, Kadzoev, aff. C-357/o9 PPU, pt 70; G. Cornelisse, "Casenote on C-357/o9 PPU ", Common Market Law Review, vol. 48, $\mathrm{n}^{\circ}$ 3, 2011, p. 925-945; M.-L. Basilien-Gainche, «De la rétention des étrangers et de ses limites dans le temps», Revue de l’Union européenne, $n^{\circ} 537$, avril 2010, p. 237-242. Il doit être mentionné ici que l'avocat général Jàn Mazák, dans sa prise de position sur l'affaire Kadzoev, a ajouté dans sa note infrapaginale 38 que «le placement en rétention, en raison d'un comportement agressif, sur un autre fondement juridique de droit national, comme, par exemple, le cas échéant, une législation destinée à préserver l'ordre public ou le droit pénal, reste toujours envisageable». Il n'en demeure pas moins qu'une telle assertion paraît difficilement compatible avec l'article 5 , $\$ 1$, de la Convention tel qu'interprété par la Cour de Strasbourg.

41. CJUE, 5 juin 2014, Bashir Mohamed Ali Mahdi, aff. C-146/14 PPU, pt 85.

42. Nations unies, "Détention d'immigrants en situation irrégulière» et "Recommandations", in Rapport du Groupe de travail sur la détention arbitraire, 16 février 2009, A/HCR/10/21, $\$ 82$.

43. Il n'existe pas de limite à la durée de la rétention au Danemark, en Irlande et au Royaume-Uni qui ont fait jouer leur opt-out concernant la directive $2008 / 115 / \mathrm{CE}$. 
appréciation mitigée que l'on peut faire de sa transposition et de son application dans les États membres: douze d'entre eux ont réduit les périodes maximales de rétention; huit les ont augmentées; treize ont choisi de se caler sur la durée maximale prévue de dix-huit mois ${ }^{44}$. Or cette dernière peut être légitimement considérée excessive ${ }^{45}$. Certes il s'agit là d'une limite absolue comme l'a martelé la Cour de Luxembourg dans son arrêt Kadzoev, qui a été l'occasion pour elle d'interpréter les dispositions de la directive « Retour»:

[...] ni l'article 15, paragraphes 5 et 6 , de la directive 2008/115 ni aucune autre disposition de cette directive ne permettent de considérer que des périodes de rétention aux fins d'éloignement ne devraient pas être incluses dans la durée maximale de rétention définie audit article 15, paragraphes 5 et 6 , en raison de la suspension de l'exécution de la décision d'éloignement ${ }^{46}$.

[...] l'article 15, paragraphe 6, de la directive 2008/115 n'autorise en aucun cas le dépassement du délai maximal défini dans cette disposition ${ }^{47}$.

Or, cette limite absolue s'accompagne d'une limite relative de la durée de la rétention aux fins d'éloignement:

[...] selon l'article 15, paragraphe 4, de la directive 2008/115, la rétention ne se justifie plus et la personne concernée est immédiatement remise en liberté lorsqu'il apparaît qu'il n'existe plus de perspective raisonnable d'éloignement pour des considérations d'ordre juridique ou autres ${ }^{48}$.

Et c'est en toute logique que la Cour de Luxembourg peut ainsi opposer aux États membres que la directive

[...] ne permet pas, lorsque la période maximale de rétention prévue par cette directive a expiré, de ne pas libérer immédiatement l'intéressé au motif qu'il n'est pas en possession de documents valides, qu'il fait preuve d'un comportement agressif et qu'il ne dispose pas de moyens de subsistance propres ni d'un logement ou de moyens fournis par l'État membre à cette fin ${ }^{49}$.

Peut-on pour autant considérer que la notion de "perspective raisonnable de retour» correspond à un retour réalisé à tout le moins avant l'expiration d'une durée de rétention de dix-huit mois? On peut en douter ${ }^{50}$. Comme l'avocat général Jàn Mazák le souligne dans sa prise de position dans l'affaire Kadzoev,
[...] la rétention d'une personne aux fins de son éloignement doit cesser dès que possible et devient illégale dès que les conditions «matérielles" de la rétention définies par cet article - notamment que le dispositif de l'éloignement est en cours et exécuté avec toute la diligence requise, et qu'il existe une perspective raisonnable d'éloignement - cessent d'être réunies ou, en tout état de cause, après l'écoulement de la durée maximale de rétention calculée selon l'article 15, paragraphes 5 et 6 , de cette directive ${ }^{51}$.

En d'autres termes, quand n'existe pas ou plus de perspective raisonnable de retour, le migrant en situation irrégulière devrait être immédiatement libéré quand bien même la durée maximale de rétention n'a pas été atteinte. Cette interprétation de la directive, que la Cour n'a pas su confirmer dans l'arrêt, semble pourtant bien la seule qui puisse être conforme à l'esprit et à la lettre de l'article 15, $\$ 1$, in fine de la directive 2008/115/CE: «Toute rétention est aussi brève que possible et n'est maintenue qu'aussi longtemps que le dispositif d'éloignement est en cours et exécuté avec toute la diligence requise». D’ailleurs la rédaction de cette disposition renvoie au septième des vingt principes directeurs sur le retour forcé adoptés par le Comité des ministres du Conseil de l'Europe le 4 mai 2005, septième principe qui concerne l' " obligation de remise en liberté en cas d'arrêt du dispositif d'éloignement » : «La détention préalable à l'éloignement ne se justifie que dans le cadre d'un dispositif d'éloignement actif»; « si le dispositif n'est pas mis en œuvre avec toute la diligence requise, la détention cesse d'être légale ${ }^{52}$. En outre, la position de l'avocat général reflète les positions que la Cour de Strasbourg a l'habitude de défendre: «seul le déroulement de la procédure d'expulsion justifie la privation de liberté fondée sur cette disposition (art. 5-1-f)» de sorte que «si la procédure n'est pas menée avec la diligence requise, la détention cesse d'être justifiée ${ }^{53}$.

En effet, la rétention aux fins d'éloignement n'est légale que dans la mesure où elle a pour objectif d'organiser et d'opérer le retour du migrant concerné quand cela est réellement possible. Cependant, il faut encore que les conditions de la rétention soient appropriées ${ }^{54}$. Selon la Commission, quatorze États membres ne sont pas à même de garantir aux migrants faisant l'objet de procédures d'éloignement qu'ils seront détenus dans des

44. Communication de la Commission au Conseil et au Parlement européen..., 28 mars 2014, COM(2014) 199, Partie IV, p. 17.

45. Rapport du rapporteur spécial sur les droits de l'homme des migrants, Jorge Bustamante, Addendum: Communications Sent to Governments and Replies Received, 20 mars 2009, A/HRC/11/7/Add.1, \$90; Conseil de l'Europe, Assemblée parlementaire, résolution 1707(2010) sur la rétention administrative des demandeurs d'asile et des migrants en situation irrégulière en Europe, 28 janvier 2010, $\$ 5$.

46. CJCE, 30 novembre 2009, Kadzoev, pt 51.

47. Ibid., pt 69 .

48. Ibid., pt 63

49. Ibid., pt 71 .

50. "The Return Directive arguably falls short of providing strong safeguards against arbitrary immigration detention» as «it fails to definitely preclude lengthy, unnecessary and disproportionate detention in the EU States» (I. Majcher, "The European Union Returns Directive...», p. 28).

51. CJCE, 30 novembre 2009, Kadzoev, pt 53.

52. Comité des ministres du Conseil de l'Europe, Vingt principes directeurs sur le retour forcé, 4 mai 2005.

53. Cour EDH, 15 novembre 1996, Chahal c. Royaume-Uni, no 22414/93, \$113. Voir aussi Cour EDH, GC, 29 janvier 2008, Saadi c. Royaume-Uni, $\$ 74 ; 19$ février 2009, A. c. Royaume-Uni, \$164;8 octobre 2009, Mikolenko c. Estonie, nº 10664/05, \$59; 27 juillet 2010, Louled Massoud c. Malte, $\mathrm{n}^{\circ} 24340 / 08, \$ 62$.

54. Cour EDH, 19 février 2009, A. c. Royaume-Uni, $\$ 164$. 
centres dédiés donc adaptés, et neuf autres ne disposent pas d'une législation nationale qui soit pleinement conforme aux exigences posées par l'article $16, \$ 1$, de la directive «Retour» concernant la séparation entre détenus migrants et détenus ordinaires ${ }^{55}$. Une question se pose ici: que doiton entendre par «centres de rétention spécialisés »? La réponse n'est pas aussi simple qu'elle pourrait sembler être: il suffit pour s'en convaincre de s'attarder sur l'étude du REM qui considère comme des «centres de rétention spécialisés » les sections dédiées des prisons en Allemagne, les centres de rétention de la police en Autriche, les antennes de police en Grèce, peu importe au demeurant que soient alors mélangés dans de mêmes espaces de confinement des migrants, des suspects, des détenus ${ }^{56}$. C'est bien pourquoi les positions affirmées par la Cour dans différents arrêts rendus le 17 juillet 2014 ont pu être regardées comme des apports majeurs. Dans sa décision dans les affaires Bero et Bouzalmate, la Cour est venue éclairer la signification de la première phrase de l'article $16, \$ 1$, de la directive 2008/115/CE:

[...] un État membre est tenu, en règle générale, de placer en rétention à des fins d'éloignement les ressortissants de pays tiers en séjour irrégulier dans un centre de rétention spécialisé de cet État ${ }^{57}$.

Par conséquent, l'utilisation de prisons autorisée par la seconde phrase de cet article $16, \$ 1$, constitue une exception, qui doit être interprétée de manière stricte. Et c'est ainsi que la Cour l'entend comme il ressort de son arrêt Pham:

[...] l'article 16, paragraphe 1 , seconde phrase, de la directive 2008/115 doit être interprété en ce sens qu'il ne permet pas à un État membre de placer en rétention à des fins d'éloignement un ressortissant de pays tiers dans un établissement pénitentiaire avec des prisonniers de droit commun même dans l'hypothèse où ce ressortissant consent à ce placement ${ }^{58}$.

Une telle interprétation de la directive "Retour» assure la conformité des normes européennes avec les standards internationaux de protection des droits de l'homme ${ }^{59}$, en ce que cette condition est appréhendée par la Cour comme une exigence non pas matérielle mais substantielle. Telle est l'idée exposée par la Cour dans l'arrêt Pham:
[...] l'obligation de séparation des ressortissants de pays tiers en séjour irrégulier des prisonniers de droit commun prévue à l'article 16 , paragraphe 1 , seconde phrase, de cette directive va au-delà d'une simple modalité d'exécution spécifique d'un placement en rétention des ressortissants de pays tiers dans des établissements pénitentiaires et constitue une condition de fond de ce placement sans laquelle, en principe, celui-ci ne serait pas conforme à ladite directive ${ }^{60}$.

Toutefois, quelles sont les implications d'une telle obligation de séparation? Une réponse à cette question est proposée par l'avocat général Yves Bot dans sa prise de position, dont on ne peut que déplorer qu'elle n'ait pas été faite sienne par la Cour:

[...] cela implique une séparation stricte des migrants des prisonniers de droit commun par l'établissement d'une unité distincte totalement isolée du reste de l'établissement pénitentiaire, n'offrant aucune possibilité de communication avec les personnes condamnées ou placées en détention avant leur jugement ${ }^{61}$.

Reste alors donc à savoir quand les autorités nationales peuvent se prévaloir de l'exception prévue au $\$ 2$ de l'article 16, autrement dit savoir quand elles peuvent recourir à des prisons. C'est encore une fois l'avocat général qui donne des éclaircissements concernant les motifs permettant de recourir pour les migrants en instance d'éloignement à la mesure la plus privative de liberté qui soit: à ses yeux, «le seul motif expressément prévu par le législateur de l'Union pour déroger à l'obligation de placement dans un centre de rétention spécialisé» ne peut correspondre qu'à «des situations d'urgence telles que celles définies à l'article 18 de la directive», qu'à des circonstances dans lesquelles un État se voit confronté à « une charge lourde et imprévue sur la capacité de ses centres de rétention ou sur son personnel administratif et judiciaire en raison d'un nombre exceptionnellement élevé de ressortissants de pays tiers soumis à une obligation de retour ${ }^{62}$.

Alors que la directive « Retour» prévoit que la rétention aux fins d'éloignement doit remplir des conditions de forme et de fond, alors même que ces conditions ont reçu de la Cour de Luxembourg des interprétations quelque peu relatives et limitées, le respect de telles garanties suppose l'exercice par les juridictions nationales d'un contrôle dont il est attendu qu'il soit intense et complet afin qu'elles puissent donner leur plein effet.

55. Communication de la Commission au Conseil et au Parlement européen..., 28 mars 2014, COM(2014) 199, Partie IV, p. 18.

56. I. Majcher, «Immigration Detention in Europe...».

57. CJUE, 17 juillet 2014, Adala Bero, aff. C-473/13 et Ettayebi Bouzalmate, aff. C-514/13, pt 32.

58. CJUE, 17 juillet 2014, Thi Ly Pham, aff. C-474/13, pt 23.

59. Pour une analyse des deux affaires mentionnées, voir I. Majcher, «The EU Returns Directive and the Use of Prisons for Detaining Migrants in Europe », EU Law Analysis, 21 juillet 2014, en ligne: http://eulawanalysis.blogspot.fr/2014/o7/the-eu-returns-directive-and-use-of.html.

60. CJUE, 17 juillet 2014, Thi Ly Pham, pt 21.

61. CJUE, 30 avril 2014, Adala Bero, aff. C-473/13 et Ettayebi Boulzamate, aff. C-514/13, conclusions de l'avocat général Yves Bot, pt 100.

62. Ibid., pt 131. Il est à noter que l'avocat général a admis un autre cas dans lequel un État membre puisse décider de la détention en prison d'un RPT en instance d'éloignement: le cas où «il existe des motifs exceptionnels et légitimes, tels que ceux qui seraient tirés de l'état de nécessité» (prise de position sur l'affaire Pham, pt 136). Reste que cette possibilité n'est pas sans inquiéter, tant il est vrai que le renvoi à des notions aussi indéfinies et aussi fluctuantes que celles d'exceptionnalité et de nécessité pose problème quand est en jeu la garantie des droits fondamentaux. 


\section{Le contrôle de la rétention aux fins d'éloignement. La vacuité de la plénitude}

Parce que la rétention des migrants en général et la rétention aux fins d'éloignement en particulier a des conséquences dommageables pour les personnes concernées en ce qu'elles accroissent les fragilités physiques et mentales existantes et qu'elles en créent de nouvelles ${ }^{6}{ }^{6}$, la vulnérabilité des migrants détenus, qui découle des traumatismes induits par leur situation pré-migratoire et de leur parcours migratoire, se trouve accentuée ${ }^{64}$. Pourtant, le confinement des migrants irréguliers échappe à un strict contrôle par le juge, alors même que l'examen de la privation de liberté est une des plus importantes missions des autorités juridictionnelles. La directive 2008/115/CE n'est en effet pas parvenue à imposer aux États membres de mettre en place un contrôle de la rétention aux fins d'éloignement qui soit tout à la fois judiciaire, préalable et obligatoire. D'abord, elle a laissé aux législations nationales le loisir de confier la décision de la rétention à une autorité judiciaire ou à une autorité administrative. Or c'est la deuxième option qui a été majoritairement adoptée par les États membres ${ }^{65}$. C’est ainsi que le juge judiciaire est compétent pour décider de la rétention des RPT en situation irrégulière aux fins d'éloignement dans sept États seulement (Allemagne, Espagne, Finlande, Irlande, Pologne, Portugal, Suède - le juge administratif ayant cette compétence en Estonie et en Lituanie), et de l'extension de la durée de rétention dans dix États membres seulement (Allemagne, Croatie, Estonie, France, Grèce, Hongrie, Irlande, Lettonie, Lituanie, Pologne). La plupart des États européens ont donc confié aux autorités administratives (officiers de police, officiers de migration et d'asile, officiers du ministère de l'Intérieur, officiers de la police aux frontières) la compétence pour appréhender les migrants en situation irrégulière et pour les placer en rétention.

Certes la directive « Retour » demande alors aux États membres d'organiser un contrôle judiciaire rapide de la rétention, ou d'offrir aux migrants détenus le droit d'introduire un recours. Or, la dernière option, évidemment moins protectrice que la première, a été privilégiée par les États membres, subordonnant l'exercice du droit au recours à l'accès des migrants détenus à une véritable assistance juridique. C'est pourquoi il n'y a pas de contrôle automatique du juge sur les décisions de mise en rétention des migrants irréguliers dans seize États membres qui ont choisi de soumettre l'exercice du contrôle à l'introduction d'un recours. L'exercice systématique d'un examen par le juge des mesures de rétention administrative aux fins d'éloignement est bien organisé dans neuf États membres (Autriche, Croatie, Estonie, Finlande, Hongrie, Lettonie, Norvège, Pays-Bas, Slovénie). Mais il est pratiqué de manière très variable selon les États: on peut noter par exemple que le contrôle a lieu tous les soixante jours en Hongrie, tous les deux mois en Lettonie, tous les trois mois en Croatie, tous les six mois en Bulgarie. Et l'on comprend sans peine pourquoi François Crépeau peut s'émouvoir et s'inquiéter des limites opposées au contrôle juridictionnel de la rétention des migrants ${ }^{66}$. Selon lui, la norme administrative, qui n'a pas intégré nombre des garanties développées dans le champ du droit pénal, est problématique en particulier en ce qui concerne la charge de la preuve et les règles de procédure; l'accès effectif au droit de recours est en réalité illusoire, dans la mesure où les migrants sont placés dans des centres de rétention qui offrent une assistance juridique grandement limitée, et une information juridique rarement disponible (dans une langue appropriée qui peut être comprise par le migrant concerné). Ainsi, alors que les autorités juridictionnelles devraient être appelées à exercer un rôle essentiel de contrôle effectif de la rétention des RPT aux fins d'éloignement, les interprétations données sur ce point par la CJUE de la directive «Retour» se révèlent malheureusement décevantes. Certes, la Cour affirme que le juge doit exercer un contrôle complet, que l'on qualifierait en droit français de plein contentieux (A). Mais elle ne va pas jusqu’à tirer toutes les conséquences logiques d'une telle affirmation, qui devraient pourtant conduire à la libération du migrant détenu (B).

\section{A. La nature du contrôle de la rétention aux fins d'éloignement. Des traits équivoques}

La CJUE, saisie de questions préjudicielles, s'est à plusieurs reprises intéressée à la nature du contrôle que l'autorité juridictionnelle a à exercer sur la rétention aux fins d'éloignement. C'est ainsi qu'elle a affirmé, dans l'affaire Arslan, que le maintien en rétention ne peut être décidé qu' «à la suite d'une appréciation au cas par cas de l'ensemble des circonstances pertinentes ${ }^{67}$, ce qui devrait aller sans dire mais qu' il lui a bien fallu rappeler. L'affaire Mahdi a offert, quant à elle, à la Cour de Luxembourg l'opportunité de donner quelques éclairages sur la portée de ce contrôle ${ }^{68}$. Aux yeux du juge de l'Union, l'autorité juridictionnelle doit d'abord étudier les décisions de placement en rétention

63. Pour une exposition des effets délétères de la détention sur les migrants d'un point de vue personnel et social, voir Jesuit Refugee Service, Europe: Becoming Vulnerable in Detention, The DEVAS Project, juin 2011.

64. Sur la question des vulnérabilités cumulées, voir M.-L. Basilien-Gainche, «Le temps des vulnérabilités», in Le monde émergent, vol. II, Pour un monde habitable: la Terre-sol, Y. C. Zarka (dir.), Paris, A. Colin, 2014, p. 93-113.

65. REM, The Use of Detention and Alternatives to Detention..., p. 24-25 et 44-45.

66. F. Crépeau, UN Special Rapporteur on the Human Rights of Migrants. FRA-ECtHR Seminar on European Law on Asylum, Borders and Immigration: Concluding Remarks, 11 juin 2013

67. CJUE, 30 mai 2013, Arslan, pt 63.

68. CJUE, 5 juin 2014, Bashir Mohamed Ali Mahdi. 
au regard de «tous» les éléments de fait et de droit du cas considéré: en d'autres termes, elle a à tenir compte non seulement des « faits et [d] es preuves produits par l'autorité administrative l'ayant saisie ", mais également à prendre en considération «les faits, les preuves et les observations qui lui sont éventuellement soumis lors de cette procédure» par le migrant détenu ou par les associations intervenantes ${ }^{69}$. Ensuite, l'autorité juridictionnelle doit exercer un contrôle qui lui permette « de statuer sur le fond, au cas par cas, sur la prolongation de la rétention du ressortissant concerné, sur la possibilité de substituer à la rétention une mesure moins coercitive ou sur la remise en liberté de ce ressortissant $\gg^{70}$. Si la CJUE estime de la sorte nécessaire que le juge exerce sur les mesures de placement en rétention aux fins d'éloignement un contrôle de nature approfondie ayant une pleine portée, elle développe par ailleurs des positions qui révèlent combien ce contrôle appelé à être fort peut être faible. La manière dont la Cour de Luxembourg envisage les atteintes au droit d'être entendu durant les procédures d'éloignement est sur ce point révélatrice ${ }^{71}$ : en vidant ce droit de sa substance et en limitant les effets du constat de sa violation, la CJUE rend de manière pour le moins paradoxale allégé le contrôle approfondi, et vide le plein contentieux.

Alors que la directive "Retour» n'énonce pas de procédure spécifique d'audition du migrant en situation irrégulière avant l'adoption à son encontre d'une mesure d'éloignement, la CJUE reconnaît l'importance de garantir aux RPT faisant l'objet de procédures de retour leur droit à être entendu, dans ses décisions Mukarubega et Boudjlida $^{72}$. La raison d'une telle assertion ne doit pas être trouvée dans l'article $41, \$ 2$, de la Charte des droits fondamentaux de l'Union européenne sur le droit à une bonne administration, comme le soutenait l'avocat général Melchior Wathelet dans sa prise de position sur les deux affaires: elle résulte des droits de la défense qui sont énoncés aux articles 47 et 48 de la Charte et qui constituent un "principe fondamental du droit de l'Union européenne ${ }^{73}$.
La consécration du droit d'être entendu peut sembler d'autant plus claire et forte que l'adjectif employé pour qualifier le principe de référence n'est pas «général » mais «fondamental». Il reste qu'une telle consécration s'avère à la lecture des arrêts de la Cour purement déclaratoire, tant la définition donnée du droit d'être entendu déçoit qui le vide de toute sa substance. Il suffit pour s'en convaincre de se reporter à la décision Boudjlida qui montre combien est limité le droit d'être entendu. Selon la Cour qui a suivi en cela la prise de position de l'avocat général,

[...] le droit d'être entendu dans toute procédure, tel qu'il s'applique dans le cadre de la directive 2008/115 et, notamment, de l'article 6 de celle-ci, doit être interprété en ce sens qu'il n'oblige l'autorité nationale compétente ni à prévenir ce ressortissant, préalablement à l'audition organisée en vue de ladite adoption, de ce qu'elle envisage d'adopter à son égard une décision de retour, ni à lui communiquer les éléments sur lesquels elle entend fonder celle-ci, ni à lui laisser un délai de réflexion avant de recueillir ses observations [...].

Et « en ce sens qu'il n'impose pas aux États membres de prendre en charge cette assistance dans le cadre de l'aide juridique gratuite $»^{74}$.

Si le droit d'être entendu ainsi compris apparaît de la sorte bien peu consistant, il se révèle par ailleurs peu disposé à revêtir une quelconque effectivité: il se réduit à « un droit fictionnel ou purement virtuel pour la plupart des étrangers en instance d'éloignement ", à " un droit d'être entendu inaudible» ${ }^{75}$. Il est en effet à souligner que, dans son appréhension des affaires Mukarubega et Boudjlida, le juge de l'Union n'a guère prêté attention aux circonstances de fait qui ont caractérisé les procédures administratives en cause. Alors que les considérations procédurales sont souvent les seuls vecteurs d'une garantie effective des droits comme le met en évidence Hiroshi Motomura ${ }^{76}$, la CJUE a négligé des éléments essentiels, révélant soit un désintérêt préoccupant pour les situations concrètes dans lesquelles les migrants en situation irrégulière se trouvent,

69. CJUE, 5 juin 2014, Bashir Mohamed Ali Mahdi, pt 64.

70. Ibid., pt 53 .

71. N. Lepoutre, «Le droit d'être entendu avant l'adoption d'une mesure d'éloignement: un droit fondamental réduit à portion congrue», Lettre «Actualités Droits-Libertés» de La revue des droits de l'homme, 6 janvier 2015, en ligne: http://revdh.revues.org/1042.

72. CJUE, $5^{\mathrm{e}}$ ch., 5 novembre 2014, Sophie Mukarubega c. préfet de police et préfet de la Seine-Saint-Denis, aff. C-166/13, et 11 décembre 2014, Khaled Boudjlida c. préfet des Pyrénées-Atlantiques, aff. C-249/13. Voir M.-L. Basilien-Gainche, T. Racho, "Quand le souci d'efficacité de l'éloignement l'emporte sur l'application effective des droits fondamentaux», Lettre "Actualités Droits-Libertés » de La revue des droits de l'homme, 18 novembre 2014, en ligne: http://revdh.revues.org/957. Il convient de noter ici que le Conseil d'État n'a pas attendu que la Cour de Luxembourg ait répondu aux questions préjudicielles qui lui étaient posées respectivement par le tribunal administratif de Melun et par le tribunal administratif de Bayonne. Il a exposé sa propre position sur ces questions dans un avis qui semble avoir grandement inspiré la CJUE (CE, 4 juin 2014, $M^{m e} H_{a l i f a}$, $\mathrm{n}^{\circ}$ 370515): le droit d'être entendu n'impose pas aux autorités administratives de donner au RPT l'opportunité de présenter ses propres observations concernant les mesures d'éloignement dont il fait l'objet, dans la mesure où il a été mis à même d'être entendu avant l'adoption de la mesure administrative qui lui a refusé un droit au séjour.

73. Arrêt Sophie Mukarubega c. préfet de police et préfet de la Seine-Saint-Denis, pt 42, qui renvoie aux décisions de la CJCE du 18 décembre 2008 , Sopropé, aff. C-349/o7, de la CJUE du 22 novembre 2012, M. M., aff. C-277/11, et du 3 juillet 2014, Kamino International Logistics BV, aff. C-129/13 et Datema Hellmann Worldwide Logistics BV, aff. C-13o/13; arrêt Khaled Boudjlida c. préfet des Pyrénées-Atlantiques, pt 30, qui renvoie aux décisions de la CJUE du 3 juillet 2014, Kamino International Logistics BV, et du 5 novembre 2014, Sophie Mukarubega c. préfet de police et préfet de la Seine-Saint-Denis.

74. CJUE, $5^{\mathrm{e}}$ ch., 11 décembre 2014, Khaled Boudjlida c. préfet des Pyrénées-Atlantiques, pts 69 et 71 respectivement.

75. S. Slama, "Dissonances et convergences jurisprudentielles sur l'étendue et la teneur du droit d'être entendu avant une décision de retour", Lexbase Hebdo édition publique, $\mathrm{n}^{\circ} 358,15$ janvier 2015.

76. H. Motomura, «The Curious Evolution of Immigration Law: Procedural Surrogates for Substantive Constitutional Rights», Columbia Law Review, vol. 92, nº 7, novembre 1992, p. 1626-1704. 
soit une incompréhension inquiétante du système français de l'immigration et de l'asile ${ }^{77}$. Or, il ne faudrait pas oublier que les droits de la défense, dont découle le droit d'être entendu, alors même qu'ils sont érigés en "principe fondamental du droit de l'Union européenne", «n'apparaissent pas comme des prérogatives absolues, mais peuvent comporter des restrictions dans certaines circonstances» comme l'a rappelé l'avocat général dans ses conclusions sur les deux affaires ${ }^{78}$. Cependant, le droit d'être entendu dont on a vu que la substance est évanescente et la portée relative, s'effondre sous le coup que lui a porté celle-là même qui l'avait auparavant consacré. Pour le juge de l'Union en effet, la violation du droit d'être entendu doit être sanctionnée uniquement si le résultat de la procédure administrative eut été différent si le migrant avait bien été entendu. Dans sa décision G. et R., la CJUE affirme ainsi que

[...] lorsque la prolongation d'une mesure de rétention a été décidée dans le cadre d'une procédure administrative en méconnaissance du droit d'être entendu, le juge national chargé de l'appréciation de la légalité de cette décision ne saurait accorder la levée de la mesure de rétention que s'il considère, eu égard à l'ensemble des circonstances de fait et de droit de chaque cas d'espèce, que cette violation a effectivement privé celui qui l'invoque de la possibilité de mieux faire valoir sa défense dans une mesure telle que cette procédure administrative aurait pu aboutir à un résultat différent ${ }^{79}$.

L'effet utile de la directive «Retour» doit prévaloir; l'efficacité des mesures d'éloignement doit primer.

Telle est la position défendue par la Cour dans sa décision Mukarubega:

[...] le droit d'être entendu dans toute procédure, tel qu'il s'applique dans le cadre de la directive 2008/115 et, notamment, de l'article 6 de celle-ci, doit être interprété en ce sens qu'il ne s'oppose pas à ce qu'une autorité nationale n'entende pas le ressortissant d'un pays tiers spécifiquement au sujet d'une décision de retour lorsque, après avoir constaté le caractère irrégulier de son séjour sur le territoire national à l'issue d'une procédure ayant pleinement respecté son droit d'être entendu, elle envisage de prendre à son égard une telle décision, que cette décision de retour soit consécutive ou non à un refus de titre de séjour ${ }^{80}$.
La raison tient manifestement au souci qu'a la Cour de prévenir les détournements de procédures: l' «obligation de l'entendre spécifiquement au sujet de la décision de retour avant d'adopter ladite décision prolongerait la procédure administrative inutilement, sans accroître la protection juridique de l'intéressée ${ }^{81}$; «le droit d'être entendu avant l'adoption d'une décision de retour ne peut être instrumentalisé pour rouvrir indéfiniment la procédure administrative ${ }^{82}$. Il s'agit là, aux yeux du juge de l'Union européenne, de limitations du droit d'être entendu qui «répondent effectivement à des objectifs d'intérêt général» et qui «ne constituent pas, au regard du but poursuivi, une intervention démesurée et intolérable qui porterait atteinte à la substance même des droits ainsi garantis ${ }^{8}$. C'est à juste titre que l'on peut, doit, se demander de quels objectifs d'intérêt général il est question ici. La réponse est fournie sans ambages par la Cour qui met en balance d'une part «le droit fondamental de l'intéressé d'être entendu avant l'adoption d'une décision lui faisant grief» et d'autre part «l'obligation des États membres de lutter contre l'immigration illégale ${ }^{84}$. En d'autres termes, il est affirmé implicitement par la CJUE que restreindre ainsi la portée du droit d'être entendu est nécessaire à la lutte contre l'immigration illégale, et proportionné à la réalisation de cet objectif. Et l'on ne peut que déplorer que la Cour de Luxembourg consacre ce faisant la lutte contre l'immigration irrégulière en l'érigeant en «objectif d'intérêt général », et valide incidemment les mécanismes légaux de création de l'immigration irrégulière ${ }^{85}$.

\section{B. Le terme de la rétention aux fins d'éloignement. Des limbes illégitimes}

Si les interprétations de la directive 2008/115/CE que la CJUE a données concernant la rétention aux fins d'éloignement peuvent décevoir, c'est en grande partie parce qu'elles ne tirent pas toutes les conséquences raisonnables et rationnelles des dispositions de ce texte. Une fois que les décisions relatives aux procédures de retour ont été sanctionnées par les juridictions nationales (mesure d'éloignement, placement en rétention aux fins d'éloignement), une fois que la période maximale de rétention de dix-huit mois a été atteinte ou que toute perspective raisonnable

77. Voir M.-L. Basilien-Gainche, «Removal Orders and Right to be Heard. The CJEU Fails to Seize how the French Asylum and Migration System is Dysfunctional ", EU Law Analysis, 12 décembre 2014, en ligne: http://eulawanalysis.blogspot.fr/2014/12/removal-orders-and-right-to-be-heard.html; S. Slama, «Dissonances et convergences jurisprudentielles...».

78. CJUE, 25 juin 2014, Sophie Mukarubega c. préfet de police et préfet de la Seine-Saint-Denis, aff. C-166/13 et Khaled Boudjlida c. préfet des PyrénéesAtlantiques, aff. C-249/13, conclusions de l'avocat général Melchior Wathelet, pts 66 et 57 respectivement.

79. CJUE, 10 septembre 2013 , G. et R., aff. C-383/13 PPU, pt 45.

80. CJUE, $5^{\mathrm{e}}$ ch., 5 novembre 2014, Sophie Mukarubega c. préfet de police et préfet de la Seine-Saint-Denis, pt 82 (nous soulignons).

81. Ibid., pt 70 (nous soulignons).

82. Ibid., pt 71 (nous soulignons).

83. Ibid., pt 53 .

84. Ibid., pt 71 .

85. Voir N. de Genova, "The Legal Production of Mexican/ Migrant Illegality», Latino Studies, vol. 2, n² 2, 2004, p. 160-185; N. de Genova, B. Neilson, W. Walters, «Foucault, migrazioni e confini», Materiali Foucaultiani, vol. 2, nº 3, 2013, p. 149-213. 
d'éloignement n'existe plus, la situation des migrants en situation irrégulière qui ne peuvent pas être éloignés se révèle incertaine, indéterminée: elle demeure largement en dehors du champ d'application des normes européennes en matière d'immigration et d'asile. Comme ces RPT ne peuvent être ni éloignés ni régularisés, ils restent sans statut, rien ne les préservant d'être à nouveau l'objet de mesures d'éloignement et de placement en rétention ${ }^{86}$. Ils sont prisonniers dans une twilight zone, dans un purgatoire légal, avec un accès si ce n'est existant à tout le moins très limité à leurs droits ${ }^{87}$. Ainsi, dans l'affaire Kadzoev, la Cour a insisté sur la nécessité pour les États membres, "lorsque la période maximale de rétention prévue par cette directive a expiré», de relâcher immédiatement un RPT, peu importe

[...] qu'il [ne soit] pas en possession de documents valides, qu'il [fasse] preuve d'un comportement agressif et qu'il ne dispose pas de moyens de subsistance propres ni d'un logement ou de moyens fournis par l'État membre à cette fin ${ }^{88}$.

Toutefois, cette assertion ne conduit pas la Cour à imposer aux États membres de délivrer au migrant concerné un permis de séjour autonome, ou bien une autorisation leur accordant le droit de rester sur le territoire national, alors même qu'il n'existe manifestement pas ou plus de perspective raisonnable de retour. C'est ainsi que les migrants en situation irrégulière restent dans des limbes certes légaux et néanmoins illégitimes que les normes ont elles-mêmes créés et nourris ${ }^{89}$.

C'est une position identique que la CJUE a adoptée dans l'arrêt Mahdi, ce qui la conduit finalement à se retrancher derrière le considérant 12 et l'article $6, \$ 4$, de la directive 2008/115/CE : elle se contente de rappeler que «les États membres peuvent décider d'accorder un titre de séjour autonome ou une autre autorisation conférant un droit de séjour pour des motifs charitables, humanitaires ou autres», laissant aux autorités nationales une large marge d'appréciation quand ce n'est pas de discrétion ${ }^{90}$. La Cour de Luxembourg n'adopte donc pas la position que la Commission semble bien avoir défendue dès le début des négociations de la directive «Retour»: la norme de l'Union devrait proposer une alternative entre l'éloignement des migrants en situation irrégulière et l'octroi d'une autorisation de séjour. Dans sa communication sur la politique européenne de retour, la Commission insiste sur la nécessité de garantir que les RPT qui ne peuvent pas être éloignés ne soient pas indéfiniment délaissés, sans accès à leurs droits, et sans protection contre le risque d'être détenus de nouveau cette fois illégalement ${ }^{91}$. Reste que cette assertion juridique, qui seule paraît logique, se heurte à des considérations éminemment politiques. Certes le groupe de contact directive "Retour» a manifesté son accord sur la nécessité de « réduire les zones grises», dans la mesure où un vide est laissé par la directive 2008/115/CE: le texte, en effet, ne traite pas des cas dans lesquels les États membres adoptent des mesures de retour qui ne peuvent pas être mises en œuvre en raison des obstacles juridiques ou pratiques s'opposant à la réalisation de l'éloignement de l'étranger concerné (manque de coopération des autorités nationales des États tiers, difficultés dans l'obtention auprès des autorités nationales des États tiers des documents permettant le voyage retour du RPT, incidences du principe de non-refoulement, etc.) ${ }^{92}$. Toutefois, sont pour le moins inquiétantes les réflexions sur les meilleures pratiques qui sont développées par le groupe de contact au sujet de la discipline commune à définir pour tous les États membres concernant le traitement des personnes non éloignables («unremovable returnees»).

De manière significative, le groupe de contact sur la directive «Retour» se montre particulièrement soucieux de prévenir que ne soient adoptées des mesures nationales permissives qui pourraient apparaître comme des récompenses à l'irrégularité et comme des facteurs d'immigration irrégulière. En d'autres termes, si «la possibilité de procéder à des régularisations individuelles basées sur des critères équitables et transparents » est admise, «les régularisations massives et systématiques de personnes en séjour irrégulier devraient être évitées", comme la Commission le déclare dans sa communication sur la politique commune d'immigration en Europe ${ }^{93}$. Or, il est à souligner que le groupe de contact se rapporte à l'étude réalisée en 2010 par Ramboll et EurAsylum pour le compte de la Commission sur la situation des ressortissants de pays qui font l'objet de mesures de retour dont l'exécution est retardée ${ }^{94}$, et qu'il utilise les propositions faites aux migrants qui ont fait l'objet de mesures de retour et qui ne peuvent être éloignés, alors que les deux situations considérées sont pour le moins

86. Voir A. Bathily, Immigration Detention and its Impact on Integration. A European Approach, KING Project - EU Policy Unit, Desk Research Paper $n^{\circ}$ 2, juillet 2014

87. Sur la twilight zone, qui concerne les étrangers «ni expulsables - ni régularisés», qui concerne certainement plusieurs centaines de milliers d'étrangers en Europe, voir S. Slama, «Directive "retour": la Cour de justice remodèle le contrôle juridictionnel et les modalités de prolongation de la rétention », Lexbase Hebdo édition publique, no 340, 17 juillet 2014

88. CJCE, 30 novembre 2009, Kadzoev, pt 71.

89. Voir C. Costello, «Human Rights and the Elusive Universal Subject», p. 303

90. CJUE, 5 juin 2014, Bashir Mohamed Ali Mahdi, pt 88.

91. Communication de la Commission au Conseil et au Parlement européen..., 28 mars 2014, COM(2014) 199.

92. Groupe de contact directive « Retour», 21 mars 2013, Migrapol CC Return Dir 50.

93. Communication de la Commission au Parlement européen, au Conseil, Comité économique et social européen et au Comité des régions, 17 juin 2008, $\operatorname{COM}(2008)$ 359, p. 15.

94. Ramboll et EurAsylum, Study on the Situation of Third-Country Nationals Pending Postponed Return/Removal in the EU Member States and the Schengen Associated Countries, 2010, HOME/2010/RFXX/PR/1001. 
différentes. Parmi les propositions avancées par l'étude de Ramboll et EurAsylum, le groupe de contact se rapporte notamment à celle qui consisterait à opérer une catégorisation des migrants faisant l'objet d'une mesure de retour: la différenciation serait à faire entre ceux qui coopèrent avec les autorités nationales et ceux qui ne coopèrent pas, afin de déterminer les mesures à prendre par la suite. Et le groupe de contact de soulever des questions toutes fort inquiétantes: il conviendrait de savoir quels droits fondamentaux devraient être ouverts aux RPT quelle que soit la catégorie dont ils relèveraient; quels droits supplémentaires devraient être reconnus aux migrants qui coopèrent; si une possibilité de régularisation devrait ou non pouvoir être ouverte aux migrants en cause, et si oui auxquels; si les États membres devraient être libres de placer de nouveau en rétention les migrants qui ont été remis en liberté après l'expiration de la durée maximale de rétention ou à raison de l'absence de perspective raisonnable de retour; si devrait être envisagée la possibilité d'emprisonner les migrants ne coopérant pas en application de la loi pénale, une fois expirée la période de rétention maximale de dix-huit mois. Si les questions posées sont préoccupantes, les réponses proposées le sont plus encore.

Pour le groupe de contact sur la directive «Retour», les États membres devraient pouvoir mettre en détention les migrants en situation irrégulière qui ont fait l'objet de mesures de retour et qui n'ont pas pu être éloignés en application du droit pénal, après leur mise en liberté à la fin de la période de rétention aux fins d'éloignement, dans la mesure où la rétention aux fins d'éloignement serait alors elle-même illégale. La mise en détention pour pallier l'impossibilité de placer ou maintenir en rétention, telle est donc la solution avancée. En effet, comme le rappelle le groupe de contact, la CJUE a considéré, dans son arrêt El Dridi, qu'

[...] une réglementation d'un État membre [...] qui prévoit l'infliction d'une peine d'emprisonnement à un ressortissant d'un pays tiers en séjour irrégulier pour le seul motif que celui-ci demeure, en violation d'un ordre de quitter le territoire de cet État dans un délai déterminé, sur ledit territoire sans motif justifié est contraire à l'article 15 de la directive "Retour» ${ }^{95}$. Or, le positionnement de la Cour s'est trouvé nuancé dans la décision Achughbabian, où il est soutenu que

[...] la directive 2008/115 ne s'oppose pas à ce que des sanctions pénales soient infligées, suivant les règles nationales de procédure pénale, à des ressortissants de pays tiers auxquels la procédure de retour établie par cette directive a été appliquée et qui séjournent irrégulièrement sur le territoire d'un État membre sans qu'existe un motif justifié de non-retour ${ }^{96}$.

Dans la mesure où la Cour ne définit pas ce qu'elle entend par "un motif justifié de non-retour», il est à craindre que les États membres puissent user voire abuser de la criminalisation de l'immigration pour gérer les flux migratoires et pour organiser la rétention des migrants irréguliers, sans tenir compte du fait que la privation de liberté soulève des questions juridiques sensibles en matière de respect des droits fondamentaux des personnes considérées ${ }^{97}$. En admettant ainsi la pénalisation de l'entrée et du séjour irréguliers comme cela ressort clairement de la décision Sagor ${ }^{98}$, le juge de l'Union valide une telle criminalisation de l'immigration irrégulière, alors que celle-ci a été maintes fois critiquée: elle affecte tous les migrants en situation irrégulière y compris les demandeurs d'asile qui se trouvent alors dans l'impossibilité de se prévaloir de leur droit à solliciter la protection internationale ${ }^{99}$.

Le problème des "unremovable returnees", que l'on ne saurait négliger, révèle ce que Matthew Gibney appelle un «deportation gap», autrement dit un hiatus entre le nombre de migrants en situation irrégulière qui font l'objet d'une mesure de retour et le nombre de ceux qui sont effectivement éloignés ${ }^{100}$. Selon les données publiées par la Commission, seuls 178 ooo des 484 ooo RPT sous le coup de mesures de reconduite à la frontière ont effectivement quitté le territoire de l'Union en 2012, tant et si bien que quelque 306000 "unremovable returnees» sont venus s'ajouter à la masse des migrants qui peuplent les limbes de l'Europe ${ }^{101}$. Dans la mesure même où le retour des étrangers en situation irrégulière est l'objectif de la rétention aux

95. CJUE, $1^{\text {re }}$ ch., 28 avril 2011, El Dridi, aff. C-61/11 PPU, pt 62. Voir M.-L. Basilien-Gainche, «Directive "retour": la Cour de Luxembourg met en cause la pénalisation de l'irrégularité entravant l'efficacité du droit de l'UE», Combats pour les droits de l'homme, 29 avril 2011, en ligne: http://combatsdroitshomme.blog.lemonde.fr/2011/04/29; M.-L. Basilien-Gainche, S. Slama, "L'arrêt El Dridi: la nécessaire remise à plat du dispositif de pénalisation de l'irrégularité », Actualité juridique. Pénal, no 7-8, juillet-août 2011, p. 362-367.

96. CJUE, 6 décembre 2009, Achughbabian, aff. C-329/11, pt 48.

97. Voir I. Majcher, "Crimmigration" in the European Union through the Lens of Immigration Detention", Global Detention Project, Working Paper no 6, 2013; V. Mitsilegas, The Criminalisation of Migration in Europe. Challenges for Human Rights and the Rule of Law, Cham, Springer, 2015; M. Provera, The Criminalisation of Irregular Migration in the European Union, Centre for European Policy Studies, 2015.

98. CJUE, 6 décembre 2012, Md Sagor. Voir M.-L. Basilien-Gainche, «Pénalisation du séjour irrégulier...».

99. Voir le rapport réalisé en 2010 sous la direction de E. Guild pour le commissaire aux droits de l’homme du Conseil de l'Europe, La criminalisation des migrations en Europe: quelles incidences pour les droits de l'homme?; le rapport publié en 2013 par le rapporteur spécial des Nations unies sur les droits des migrants, F. Crépeau, Étude régionale: la gestion des frontières extérieures de l'Union européenne et ses incidences sur les droits de l'homme des migrants; les rapports de l'Agence des droits fondamentaux de l'Union européenne qui ont été publiés en novembre 2011, Les droits fondamentaux des migrants en situation irrégulière dans l'Union européenne, en mars 2013, Les droits fondamentaux aux frontières maritimes méridionales de l'Europe, et en mars 2014, Pénalisation des migrants en situation irrégulière et des personnes qui les aident. Voir en outre M.-L. Basilien-Gainche, "Leave and Let Die. The EU Banopticon Approach of Migrants at Sea", in Boats Refugees and Migrants at sea. A Comprehensive Approach Integrating Maritime Security with Human Rights, V. Moreno-Lax, E. Papastavridis (dir.), Leyde, Brill, 2015.

100. M. J. Gibney, «Asylum and the Expansion of Deportation in the United Kingdom », Government and Opposition, vol. 43, n 2, 2008, p. 146-167.

101. Communication de la Commission au Conseil et au Parlement européen..., 28 mars 2014, COM(2014) 199. 
fins d'éloignement en vertu de la directive 2008/115/CE, le mécanisme doit être questionné tant son efficacité est relative. Si les taux de retour sont si bas, c'est que différents obstacles sont rencontrés par les États membres dans la mise en œuvre des reconduites à la frontière. Il s'agit d'abord de problèmes administratifs, qui tiennent pour beaucoup au manque de coopération des autorités nationales des États tiers, en particulier aux difficultés opposées par les autorités de l'État tiers dont le RPT est le ressortissant à la délivrance des pièces nécessaires à la réalisation de son retour. Il s'agit ensuite et surtout des obligations juridiques, qui découlent des droits fondamentaux que les États ont à garantir en application des normes internationales, européennes, nationales (droit à une assistance médicale, droit au regroupement familial, droit de l'asile). Face aux lourds investissements et importantes dépenses qui ont été consentis pour promouvoir la rétention des migrants irréguliers, la politique de retour du fait de sa manifeste inefficacité révèle son défaut de rationalité, comme le mettent en exergue à juste titre Arjen Leerkes et Dennis Broeders ${ }^{102}$. Derrière un tel paradoxe, certains discernent les fonctions sociales que l'Union européenne et les États membres font remplir à la rétention aux fins d'éloignement: d'abord, prévenir l'immigration irrégulière en dissuadant les RPT de s'aventurer vers le mirage de l'Europe; ensuite, gérer des populations marginalisées en les marginalisant plus encore; enfin, afficher devant les opinions publiques nationales la souveraineté d'États impliqués dans le contrôle strict de leurs frontières ${ }^{103}$. Au-delà du constat du défaut d'efficacité et de rationalité de la politique de l'Europe en matière de retour et de rétention des étrangers en situation irrégulière, il conviendrait d'apprécier une telle politique à l'aune des principes de nécessité et de proportionnalité que la CJUE a l'habitude d'employer dans ses raisonnements. En effet, la politique de retour et de rétention n'hésite pas à permettre que soient violés les droits fondamentaux de milliers de migrants en situation irrégulière, qu'elle a elle-même contribué à créer, en les délaissant dans des limbes qui pour être légaux n'en sont pas moins illégitimes ${ }^{104}$.

102. A. Leerkes, D. Broeders, "A Case of Mixed Motives? Formal and Informal Functions of Administrative Immigration Detention", British Journal of Criminology, vol. 50, $\mathrm{n}^{\circ} 5,2010$, p. 836.

103. Voir M. Vanderbruggen, J. Phelps, N. Sebtaoui, A. Kovats, K. Pollet, Point of No Return. The Futile Detention of Unreturnable Migrants, Bruxelles, Flemish Refugee Action, 2014.

104. Voir A. Edwards, Back to Basics: The Right to Liberty and Security of Person and "Alternatives to Detention" of Refugees, Asylum-Seekers, Stateless Persons and Other Migrants, Genève, UNHCR, 2011, PPLA/2011/o1.Rev.1. 